\title{
Emergent propagation modes of ferromagnetic swimmers in constrained geometries
}

M.T. Bryan, S.R. Shelley, M.J. Parish, P.G. Petrov, C.P. Winlove, A.D. Gilbert, F.Y. Ogrin

College of Engineering, Mathematics and Physical Sciences, University of Exeter, Exeter, EX4 4QL

Magnetic microswimmers, composed of hard and soft ferromagnets connected by an elastic spring, are modelled under low Reynolds number conditions in the presence of geometrical boundaries. Approaching a surface, the magneto-elastic swimmer's velocity increases and its trajectory bends parallel to the surface contour. Further confinement to form a planar channel generates new propagation modes as the channel width narrows, altering the magneto-elastic swimmer's speed, orientation and direction of travel. Our results demonstrate that constricted geometric environments, such as occur in microfluidic channels or blood vessels, may influence the functionality of magnetoelastic microswimmers for applications such as drug delivery. 


\section{INTRODUCTION}

Modern fabrication techniques make it feasible to manufacture structures of similar scale to biological cells and subcellular structures. This has inspired a drive to produce microbots capable of entering the body to perform useful therapeutic functions, such as microdrilling ${ }^{1}$ or directing drug delivery to locally increase concentration in target sites while limiting off-target dosage elsewhere, therefore reducing side effects. ${ }^{2}$ Additionally, the potential of microbots for cell stimulation ${ }^{3}$ and transportation ${ }^{4}$ have made them desirable for chip-based assays and microfluidic applications. Key to the achieving this goal is the ability to create directed movement through a fluid. However this is not trivial, as motion at micrometre-sized dimensions is dominated by the effect of viscous forces, characterised by a low Reynolds number. In the low Reynolds number regime, inertial effects are negligible, so a swimmer in a Newtonian fluid cannot generate net motion if the deformation attempting to drive propulsion is reciprocal under time-reversal. ${ }^{5}$ Therefore, the challenge has been to design low Reynolds number swimmers capable of performing within the environment presented by the body or microfluidic devices.

While a number of propulsion methods have been proposed for generating motion under low Reynolds number conditions, ${ }^{2,6-8}$ the most promising strategy for in vivo swimming has been to use magnetic elements within the swimmer. ${ }^{9-19}$ Magnetic actuation enables propulsion to be controlled wirelessly without affecting biological viability, an essential requirement of many biomedical applications, with the added benefit that the magnetic field also determines the direction of motion. Simple magnetic attraction of particles can be effective, but requires the magnetic field to have both a strong magnitude and gradient, so this solution is only viable when the particles are close to the magnetic poles (e.g. in surface tissue). ${ }^{20,21}$ By contrast, swimming in uniform time-varying fields is possible via the coordinated movement of magnetic elements. Several groups have used helical structures that are either coated with a layer of magnetic film or which have a ferromagnetic head to generate motion in a rotating field via a screw-like mechanism. ${ }^{9-13}$ Alternatively, swimming has been demonstrated using interacting superparamagnetic particles, either to produce flagellum-like motion when the particles are physically connected ${ }^{22}$ or to produce swarm-like collective motion of individual particles. ${ }^{14-17}$

Our group proposed a third strategy based on two ferromagnetic particles with differing size and magnetic anisotropies, connected by an elastic link. ${ }^{18,19}$ Experimental demonstration of a prototype magneto-elastic swimmer can be found in the supplementary material of reference 19 . This breaks time-reversal symmetry via the combined actions of the structural asymmetry, the torque induced by an oscillating applied field and of the interaction between the ferromagnetic particles, which alternates between repulsion and attraction due to reversal of the magnetically softer particle during the field cycle. By decoupling the mechanism of propulsion from the specific shape of the swimmer, the magneto-elastic swimmer could be designed to produce different flow fields around them than those generated by corkscrew-type swimmers. ${ }^{11}$ Given that proposed drug delivery applications will involve propulsion around blood vessels, this could be advantageous since the cells lining the vasculature produce biological signalling that can either be beneficial or detrimental, depending on the specific shear stress conditions they are exposed to. ${ }^{23}$ As understanding of how physical stimuli affect biological responses is still developing, the ability to modify swimmer shape could prevent unnecessary inflammatory responses. In addition, physically linking the magnetic particles makes the 
magneto-elastic swimmer inherently more stable than swarm-type swimmers ${ }^{24}$ and therefore less likely to break up in the turbulent and divergent flow conditions that exist within the blood stream. ${ }^{25}$

Current research effort has largely concentrated on establishing structural designs capable of producing net translational motion either in purely unconstrained (bulk) fluids or purely along an interface (such that the interface does not impede motion). Indeed, many swarm-type swimmers cannot propagate in bulk fluids and require the interface to generate propulsion. ${ }^{15-17}$ While necessary for establishing proof of principle for swimming, this approach has neglected to consider the effects of encountering a geometrical barrier on motion of otherwise free swimmers. However, biomedical and microfluidic applications will require swimmers initially moving in bulk fluids to interact with surface barriers, such as the cell membrane, blood vessel wall or the sides of a microfluidic chamber. Such considerations have been studied with regard to understanding biological low Reynolds swimmers, ${ }^{26,27}$ but the implications for efficiency and functionality of artificial swimmers has received little attention. Here, we demonstrate that the magneto-elastic swimmers can move either in an unconstrained fluid, near a surface or in a channel, but that the proximity of surface barriers results in changes in the swimming propagation mode. The relative orientation of the applied field axes and the surface/channel enables velocity to be enhanced and influences the region of the swimmer that interacts with the surface. If only a portion of the swimmer is used to capture cargo, such control over the surface interaction could enable a physical mechanism of programmable drugs release by postponing final delivery until the field axes are rotated.

\section{MODEL DEFINITION}

Building on our previous work, ${ }^{18,19}$ we consider a swimmer composed of two spherical ferromagnetic beads of different size joined by a spring within a rotating elliptical magnetic field (fig. 1). We assume the spring has unstrained length, $L=6.4 \mu \mathrm{m}$, and spring constant, $k_{s}=0.05 \mathrm{~N} / \mathrm{m}$, but presents no hydrodynamic drag. The field rotates clockwise at frequency $f=400 \mathrm{~Hz}$ and has major and minor axis amplitudes of $H_{a}=0.5 \mathrm{kG}$ and $H_{b}=0.01 \mathrm{kG}$, respectively. To facilitate modelling of interactions with surfaces not aligned with the local coordinate system defined by field axes, the field axes are rotated clockwise with respect to the global coordinate system by an angle, $\psi$. Therefore the $\mathrm{x}$ - and $\mathrm{y}$-fields at time $t$ in the global coordinate system are $H_{x}=H_{a} \cos (2 \pi f t) \cos \psi-H_{b} \sin (2 \pi f t) \sin \psi$ and $H_{y}=$ $-H_{a} \cos (2 \pi f t) \sin \psi-H_{b} \sin (2 \pi f t) \cos \psi$, respectively. Denoting bead number with indices, the beads have saturation magnetization, $M_{1}=M_{2}=1400 \mathrm{kA} / \mathrm{m}$; radius $R_{1}=0.8 \mu \mathrm{m}$ and $R_{2}=1.6 \mu \mathrm{m}$; and anisotropy field $H_{k 1}=100 \mathrm{kG}$ and $H_{k 2}=0 \mathrm{kG}$. Magnetization vectors $\mathbf{M}_{1}$ and $\mathbf{M}_{\mathbf{2}}$ (with polar angles, $\theta_{1}$ and $\theta_{2}$ and azimuthal angles, $\phi_{1}$ and $\phi_{2}$ ) are defined relative to the global coordinate system, as is the vector describing the axis of the spring, $\mathbf{L}\left(\theta_{s}, \phi_{s}\right)$, which coincides with the uniaxial anisotropy axis of both beads. Propagation occurs in a fluid of dynamic viscosity, $\eta,=0.1$ Pa.s, either unconstrained or bounded by one or more surfaces in the $x-z$ plane (in the global coordinate system).

Modelling propagation in a high-viscosity fluid, we apply the conditions of low Reynolds number swimming: inertial effects are neglected, such that motion in the system can be solved purely by considering the total force acting on the swimmer at any particular instance. The total force, $\mathbf{F}$, acting on the swimmer is $\mathbf{F}=\mathbf{F}_{\boldsymbol{m}}+\mathbf{F}_{\boldsymbol{E}}+\mathbf{F}_{\boldsymbol{H}}$, where $\mathbf{F}_{\boldsymbol{m}}$ is the dipolar magnetic force acting between the beads, $\mathbf{F}_{E}$ is the elastic force due to the spring and $\mathbf{F}_{H}$ is the hydrodynamic force due to the motion of 
the beads within the fluid. In addition, the magnetic field applies a torque, $\mathbf{T}$, generating a rotation of the swimmer about the centre of reaction. ${ }^{18}$

$\mathbf{T}$ and $\mathbf{F}_{\boldsymbol{m}}$ are both dependent on the effect of the magnetic field on the magnetization of each bead. We model the beads as point dipoles. Thus, for a bead $j$, the magnetic energy, $E_{j}$, is a summation of the anisotropy and Zeeman energies:

$$
E_{j}=0.5 M H_{k j}\left[1-\sin ^{2}\left(\theta_{j}-\theta_{s}\right) \cos ^{2} \varphi_{j}\right]-\mu_{0} \mathbf{M}_{j} \cdot \mathbf{H}
$$

where $j=1$ or $2, \mu_{0}$ is the permeability of free space, $\mathbf{M}_{j}$ is the magnetization vector of bead $j$ and $\mathbf{H}$ is the magnetic field vector. The polar and azimuth magnetization components can be found by computationally minimizing (1) with respect to $\theta_{j}$ and $\phi_{j}$. This enables the calculation of the torque acting on each bead, $\mathbf{T}_{j}=\mu_{0} V_{j} \mathbf{M}_{j} \times \mathbf{H}$. As the beads are linked, the net torque generates rotation of both beads about the swimmer's centre of reaction. Although the applied field is uniform, each bead experiences a non-uniform field due to the dipole field of its neighbour. The force, $\mathbf{F}_{\mathbf{m j}}$, on bead $j$ from the dipole field from bead $k, \mathbf{B}_{\mathbf{k}}$, is

$$
\mathbf{F}_{\boldsymbol{m} j}=V_{j} \nabla\left(\mathbf{M}_{\boldsymbol{j}} \cdot \mathbf{B}_{\boldsymbol{k}}\right), \quad \mathbf{B}_{\boldsymbol{k}}=\frac{\mu_{0} V_{k}}{4 \pi}\left(\frac{3\left(\mathbf{M}_{\boldsymbol{k}} \cdot \Delta \mathbf{r}\right) \Delta \mathbf{r}}{\Delta r^{5}}-\frac{\mathbf{M}_{\boldsymbol{k}}}{\Delta r^{3}}\right)
$$

where $k=3-j, V_{j, k}$ is the volume of bead $j$ or $k, \Delta r=r_{2}-r_{1}$ is the vector difference between the position vectors of beads 2 and 1 and $\Delta r$ is the magnitude of $\Delta r$.

Depending on the relative directions of the bead magnetizations, the dipolar force may cause the beads to either attract or repel. Counteracting this motion is the elastic force from the spring, which resists compression or extension from the relaxed state. Assuming the bending modulus of the spring is infinitely large, the elastic force on bead $j, \boldsymbol{F}_{E j}$, is given by

$$
\mathbf{F}_{E j}=(-1)^{j} k_{s}(\mathbf{L}-\Delta \boldsymbol{r})
$$

where $\mathbf{L}$ describes the orientation of the spring and has magnitude $L$, the unstrained spring length.

To model the hydrodynamic forces affecting the swimmer, we represent each bead as a perfect sphere and make use of the Stokes approximation, but neglect hydrodynamic drag due to the elastic spring. As each bead moves through the fluid, it not only experiences a viscous drag force proportional to its velocity, but also produces a flow that interacts with the other bead. We model the flow as uniform at the point of interaction and assume no external flow. Swimmers near a surface boundary are modelled under no-slip conditions (at the boundary there is no flow perpendicular or parallel to the surface) using the image swimmer method, which adds a mirror reflection of the swimmer on the other side of the surface to replicate the reflection of flow from the boundary surface. ${ }^{28}$ Therefore, the hydrodynamic force acting on bead $j, \mathbf{F}_{H j}$, is

$$
\mathbf{F}_{\boldsymbol{H} j}=-6 \pi \eta R_{j}\left(\mathbf{v}_{\boldsymbol{j}}-\mathbf{U}_{\boldsymbol{k}}-\mathbf{U}_{\boldsymbol{j}}^{\text {image }}-\mathbf{U}_{\boldsymbol{k}}^{\text {image }}\right)
$$

where $R_{j}$ is the radius of bead $j, \mathbf{v}_{j}$ is the velocity of bead $j, \mathbf{U}_{k}$ is the flow due to the motion of bead $k$, $\mathbf{U}_{j}^{\text {image }}$ is the flow due to the motion of the image of bead $j$ and $\mathbf{U}_{\boldsymbol{k}}^{\text {image }}$ is the flow due to the motion of the image of bead $k$. 
$\mathbf{U}_{k}$ follows the general form of the flow field from a sphere at position $\mathbf{r}_{k}$ to position $\mathbf{r}_{j}$ :

$$
\mathbf{U}_{\boldsymbol{k}}=R_{k} G^{S}\left(\mathbf{r}_{\boldsymbol{j}}-\mathbf{r}_{\boldsymbol{k}}\right) \cdot \mathbf{v}_{\boldsymbol{k}}
$$

where $R_{k}$ and $\mathbf{v}_{k}$ are the radius and velocity of bead $k$, respectively, and $G^{S}$ is a Stokeslet Greens function. For a general position vector, $r=\left(r_{x}, r_{y}, r_{z}\right)$, the Stokeslet Green's function has the form

$$
G^{S}(\mathbf{r})=\frac{3}{4 r^{3}}\left[\begin{array}{ccc}
r_{x}^{2}+r^{2} & r_{x} r_{y} & r_{x} r_{z} \\
r_{x} r_{y} & r_{y}^{2}+r^{2} & r_{y} r_{z} \\
r_{x} r_{z} & r_{y} r_{z} & r_{z}^{2}+r^{2}
\end{array}\right]
$$

As we define surface boundaries in the $x-z$ plane, the $x$ - and $z$-coordinates of the image beads are identical to the real swimmer, but the $y$-coordinates are reflected about the boundary surface. Defining the separation between the surface of the real bead and the boundary surface as $h_{j}$ (such that $h_{j}=0$ when the bead touches the boundary), the image bead will be separated from the boundary by $-h_{j}$. For example, if a boundary exists at $y=y_{\text {surf, }}$ bead $j$ at position vector $r_{j}=\left(r_{x}, y_{\text {surf }}+R_{j}+h_{j}, r_{z}\right)$ moving with velocity vector $\mathbf{v}_{j}=\left(v_{x}, v_{y}, v_{z}\right)$ will have an image at $\mathbf{r}_{j}^{\prime}=\left(r_{x}, y_{\text {surf }}-R_{j}-h_{j}, r_{z}\right)$ and velocity $\mathbf{v}_{j}{ }_{j}$ $=\left(v_{x},-v_{y}, v_{z}\right)$, such that the image moves in the opposite direction along the $y$-axis to the real swimmer. To take this into account, and to impose a no-slip condition at the boundary, we apply a modified Green's function for the image swimmer, $\mathrm{G}^{\text {image }}$, such that

$$
G^{\text {image }}(\mathbf{r}) \cdot \mathbf{v}_{\boldsymbol{j}}=G^{S}(\mathbf{r}) \cdot \mathbf{v}_{\boldsymbol{j}}-2 h_{j} G^{S t D}(\mathbf{r}) \cdot \mathbf{v}^{\prime}{ }_{\boldsymbol{j}}+2 h_{j}^{2} G^{S o D}(\mathbf{r}) \cdot \mathbf{v}^{\prime}{ }_{j}
$$

where $\mathbf{v}_{j}{ }_{j}$ is the velocity of the image of bead $j, G^{S}$ is the Stokeslet Green's function given in (6), $G^{S t D}$ is the Stokes doublet Green's function (which we reduce here to the form for a surface on the $x-z$ plane) and $G^{S O D}$ is the source doublet Green's function:

$$
\begin{array}{r}
G^{S t D}(\mathbf{r})=\frac{3}{4 r^{5}}\left[\begin{array}{ccc}
-3 r_{x}^{2} r_{y}+r_{y} r^{2} & -3 r_{x} r_{y}^{2}+r_{x} r^{2} & -3 r_{x} r_{y} r_{z} \\
-3 r_{x} r_{y}^{2}-r_{x} r^{2} & -3 r_{y}^{3}+r_{y} r^{2} & -3 r_{y}^{2} r_{z}-r_{z} r^{2} \\
-3 r_{x} r_{y} r_{z} & -3 r_{y}^{2} r_{z}+r_{z} r^{2} & -3 r_{y} r_{z}^{2}+r_{y} r^{2}
\end{array}\right] \\
G^{S O D}(\mathbf{r})=\frac{3}{4 r^{5}}\left[\begin{array}{ccc}
-3 r_{x}^{2}+r^{2} & -3 r_{x} r_{y} & -3 r_{x} r_{z} \\
-3 r_{x} r_{y} & -3 r_{y}^{2}+r^{2} & -3 r_{y} r_{z} \\
-3 r_{x} r_{z} & -3 r_{y} r_{z} & -3 r_{z}^{2}+r^{2}
\end{array}\right]
\end{array}
$$

Therefore, the flow acting on bead $j$ due to the motion of its own image is

$$
\mathbf{U}_{\boldsymbol{j}}^{\text {image }}=R_{j} G^{\text {image }}\left(\mathbf{r}_{\boldsymbol{j}}-\mathbf{r}_{\boldsymbol{j}}^{\prime}\right) \cdot \mathbf{v}_{\boldsymbol{j}}
$$

and the flow acting on bead $j$ due to the motion of the image of bead $k$ is

$$
\mathbf{U}_{\boldsymbol{k}}^{\text {image }}=R_{k} G^{\text {image }}\left(\mathbf{r}_{\boldsymbol{j}}-\mathbf{r}_{\boldsymbol{k}}^{\prime}\right) \cdot \mathbf{v}_{\boldsymbol{k}}
$$

where $\mathbf{r}_{j}{ }_{j}$ and $\mathbf{r}^{\prime}{ }_{k}$ are the position vectors of the image of beads $j$ and $k$, respectively. Where multiple boundaries are considered, a second image swimmer is introduced by adding extra terms of similar form to (10) and (11) to (4). Due to the fast decay of flow with distance, we do not consider multiple reflections when additional surfaces are added. 


\section{RESULTS AND DISCUSSION}

\section{A. Swimming constrained by a single surface}

Motion of unconstrained swimmers is characterised by a "locomotive" mode of propagation, ${ }^{29}$ in which the hard and soft beads follow circulating paths, generating net linear propagation at $15 \mu \mathrm{m} / \mathrm{s}$ in a direction perpendicular to the average orientation of the spring axis linking the beads $\left(\psi=0^{\circ}\right.$, fig. $2 \mathrm{a}$ and detail shown in Supplementary Material fig. SM1). This behaviour is replicated when a surface boundary is present, but well separated $(100 \mu \mathrm{m})$ from the swimmer (data not shown). However, as the swimmer approaches the surface, the trajectory progressively changes, such that by $5 \mu \mathrm{m}$ separation, the trajectory has altered markedly $\left(\psi=0^{\circ}\right.$, fig. $2 \mathrm{~b}$ and detail shown in Supplementary Material fig. SM2). Interactions with reflected flows from the surface repel the swimmer as it approaches the boundary, rapidly causing its motion to diverge from the path of the unconstrained system until the propagation direction stabilises parallel to the surface. This behaviour is insensitive to the initial orientation of the swimmer, since the swimmer rotates to a preferred orientation with respect to the field within the first few field cycles, well before the propagation direction stabilises. Although proximity to the boundary changes the propagation direction, the orientation of the swimmer is dependent on the angle of the elliptical field with respect to the surface $(\psi)$, so the swimmer no longer propagates perpendicular to the spring axis, but at a more acute angle (fig. $2 \mathrm{c}$ ). Since the force due to flow reflected from the boundary (from the image swimmer) is proportional to $1 / r^{3}[(8)$ and (9)], the fastest swimming occurs when the beads are closest to the boundary (at $\psi=-$ $18^{\circ}$, when the swimmer is parallel with the surface). Due to the field-dependence of the swimmer orientation, the final velocity may be reduced to $3 \mu \mathrm{m} / \mathrm{s}$ or enhanced up to $40 \mu \mathrm{m} / \mathrm{s}$ depending on $\psi$ (fig. 2c). Taken together, the change in velocity and swimmer orientation with respect to swimming direction indicate that the presence of a boundary induces a new mode of propagation that is distinct from the unconstrained case.

Repulsion of swimmers from a boundary is a consequence of hydrodynamic interactions under no-slip conditions and has been widely reported for biological swimmers. ${ }^{26,27}$ However, biological swimmers continue to face parallel to their swimming direction as they are deflected by the surface, so do not experience a change in their propagation mode. The emergence of a new propagation mode in the ferromagnetic swimmers is due to differences in propulsion mechanisms. Ferromagnetic swimmers are influenced by a combination of magnetic, hydrodynamic and elastic forces, which decouples swimmer orientation, propagation direction and velocity. By contrast, biological swimmers selfpropel through undulations of flagella or cilia, such that propagation direction is intrinsically linked to the swimmer's orientation and only determined by hydrodynamic effects. Field control over ferromagnetic swimmer orientation may provide additional functionality for applications, for example by enabling a drug-labelled region of the swimmer to be withheld from the surface until required.

\section{B. Swimming in a channel}

Lab-on-a-chip applications of magnetic swimmers require understanding of behaviour in microchannels, imposing further constraints on the boundary geometry. Therefore, planar channels were modelled by introducing extra image bead terms in (4) to describe an additional surface above the swimmer. We examined the effect of channel width and field angle on the swimmer propagation, initialising the swimmer at the centre of the channel. 
Three distinct swimming modes were observed at different channel widths and field orientations (fig. 3a). Typical manifestations of each mode can be seen by considering swimmer motion as the channel narrows under a field orientation of $\psi=90^{\circ}$ (figs. 3b-d). Details of figs. $3 \mathrm{~b}-\mathrm{d}$ are presented in Supplementary Material figs. SM3-SM5, in which traces of individual particles and the overall displacement (centre of reaction) during the last three field cycles can be resolved. For the widest channels, the swimmer propagates in the single boundary mode $(10 \mu \mathrm{m}$, fig. $3 \mathrm{~b}$ and Supplementary Material fig. SM3). This mode occurs when both beads complete tight circuits and each bead position is biased to one side of the channel throughout propagation. As (4) and (7) - (11) show the force from reflected flows decreases rapidly with distance $\left(G^{S t D}\right.$ and $G^{S O D}$ vary as $\left.1 / r^{3}\right)$, the contribution of the furthest boundary from each bead is weak, while the closest boundary dominates. Reducing the channel width inhibits the ability of the swimmer to adopt the preferred orientation to the field. Supplementary figs. SM4 and SM5 show that, although the beads may contact the channel walls in narrow channels, the beads neither penetrate nor bounce off the boundary, but instead slide along a hard surface due to the combined effect of the magnetic and elastic forces. Beyond a critical channel width, both boundaries produce significant reflected flow forces affecting both beads. The single boundary mode gradually changes into a new configuration in which the circulatory motion of the beads becomes very large and propagation occurs almost parallel with the average orientation of the spring axis linking the beads, a "pendulum" mode ( $8 \mu \mathrm{m}$, fig. $3 \mathrm{c}$ and Supplementary Material fig. SM4). Although the hard bead oscillates across the whole channel width, therefore interacting with both boundaries, the soft bead oscillates in much smaller circuits biased to a particular side of the channel. A characteristic of this mode is that the soft bead leads the propagation, a consequence of the asymmetric positioning of the soft bead, which causes it to receive a stronger, more constant force from flows reflecting of the boundaries than the hard bead. This contrasts with the final propagation mode that emerges as the channel narrows further, in which the swimmer still moves in a pendulum mode, but the hard bead leads the propagation ( $4 \mu \mathrm{m}$, fig. $3 \mathrm{~d}$ and Supplementary Material fig. SM5). Crucially, the soft bead in the hard-leading pendulum mode oscillates symmetrically about the centre of the channel. The large size of the soft bead means that it has a similar interaction with each boundary, leading to a cancelation of forces due to reflected flows. On the other hand, the smaller hard bead periodically moves much closer to one boundary than the other, resulting in less cancellation of reflected flows. On average, this means that the hard bead experiences larger forces than the soft bead and therefore drives the propagation.

Swimmer speed and direction depends on a combination of the swimming mode, channel width and field orientation (fig. 4). In general, the single boundary mode produces the slowest swimmers, although fast swimming is achieved in wide channels for $\psi=-54^{\circ}$ to $-9^{\circ}$. This asymmetry is analogous to that observed when only one surface is present (fig. 2c) and occurs due to the interaction between the field and the swimmer orientation. Increased velocity also occurs around the transition between the single boundary mode and soft-leading pendulum mode. Despite this, once swimming enters the soft-leading propagation mode, reductions in the channel width progressively reduce the velocity. In this mode, both boundaries contribute to the motion of each bead. However, since reflections from one surface partially cancel the other, the effect of reducing the channel width is to shift the soft bead driving motion towards the centre of the channel, where there is lower overall force acting on it and therefore lowering the swimming speed. Around the boundary between the soft-leading and hardleading pendulum modes the swimmer becomes almost stationary as the forces acting on each bead cancel out. In the hard-leading propagation mode, motion is driven by the transient periods when the hard bead comes close to the channel surfaces. Initially, this increases the swimming velocity as 
channel width reduces, since the narrowed channel increases the period of strong interaction. However, further narrowing below an optimum channel width results in slower swimming as restrictions to the swimmer's rotational degree of freedom begin to affect its ability to break timereversal symmetry. Nevertheless, under optimum conditions (channel width $=5.6 \mu \mathrm{m}$ and $\psi= \pm 90^{\circ}$ ), swimmers in the hard-leading pendulum mode travel at $49 \mu \mathrm{m} / \mathrm{s}$, the fastest swimming speeds in this study and almost three times faster than the unconstrained swimmer.

The presence of different propagation modes has implications for using the swimmers to deliver cargo (such as drugs or bioactive molecules) within the body or in microfluidic devices. Since the swimmers in the phase region between the hard- and soft-leading pendulum modes are almost stationary, the model indicates that swimmers may be deliberately trapped in a narrowing channel crossing the phase boundary, providing a mechanism of delivering a load to a specific area. Alternatively, since $\psi$ represents the angle between the surface and the elliptical field axes, it will change as the swimmer moves along curved channels, enabling geometric control of the propagation mode. Since the pendulum modes involve a much stronger interaction with the channel walls than the single boundary mode, geometric modal control could also provide a pathway to autonomous instigation of chemical binding.

\section{CONCLUSION}

In summary, we have developed a model describing the motion of geometrically constrained microswimmers consisting of elastically-linked ferromagnetic particles. This is of technological importance for potential applications of these swimmers, either in vivo or in microfluidic devices, where encounters with surfaces are likely to be an essential part of operation. When approaching a single surface, swimmers are progressively deflected until their direction of propagation stabilises parallel to the surface. Depending on the orientation of the elliptical applied field with respect to the surface, the final velocity may be enhanced up to $40 \mu \mathrm{m} / \mathrm{s}$, more than twice as fast as an unconstrained swimmer, or reduce down to $3 \mu \mathrm{m} / \mathrm{s}$.

When operating between two surfaces, such as within a microfluidic channel, the constraints of the channel surfaces further modify swimmer behaviour. In very wide planar channels, the swimmer only feels the presence of one surface. However, in narrower channels, the propagation mode depends on not only the channel width, but also the orientation of the elliptical field with respect to the channel. When both channel walls influence motion, the mode of swimming changes to a "pendulum" mode where part of the swimmer undergoes large oscillations across the whole of the channel width. Two distinct types of pendulum mode occur, enabling the direction of propagation to be controlled either using the channel width or the orientation of the applied field. For the magneto-elastic swimmer studied, an optimum velocity of $49 \mu \mathrm{m} / \mathrm{s}$ occurred in $5.6 \mu \mathrm{m}$ channels when the major axis of the elliptical field is applied perpendicular to the channel. While the precise optimum conditions may vary for swimmers of different size and composition, we have demonstrated that interaction with barrier or channel geometries can lead to the emergence of propagation modes that are not seen in bulk fluids and may enhance swimming velocity. Therefore, operation of magneto-elastic microswimmers in a constrained geometry may influence their functionality for potential therapeutic or microfluidic applications. 


\section{SUPPLEMENTARY MATERIAL}

See Supplementary Material for detailed enlargements of particle traces shown in figs. 2 and 3.

\section{ACKNOWLEDGEMENT}

We acknowledge the financial support from EC Contract 665440 "ABIOMATER". 


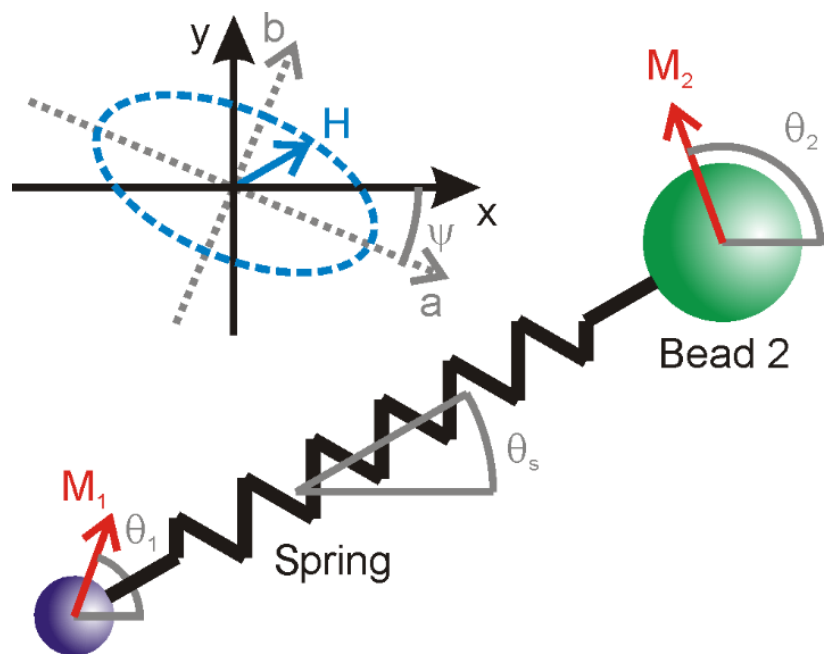

\section{Bead 1}

Figure 1: The coordinate system of the ferromagnetic swimmer and the applied elliptical field. The local coordinate system $(a, b)$ defines the major and minor axes of the clockwise rotating field, $\mathbf{H}$, which are rotated clockwise from the global coordinate axes $(x, y)$ by an angle, $\psi$. 
(a)

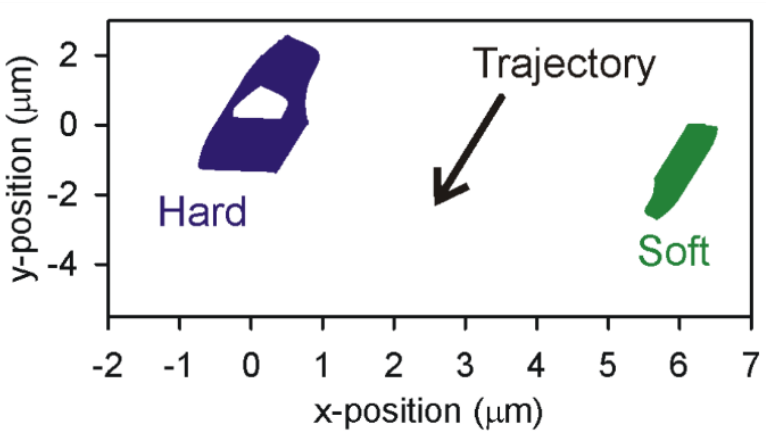

(b)
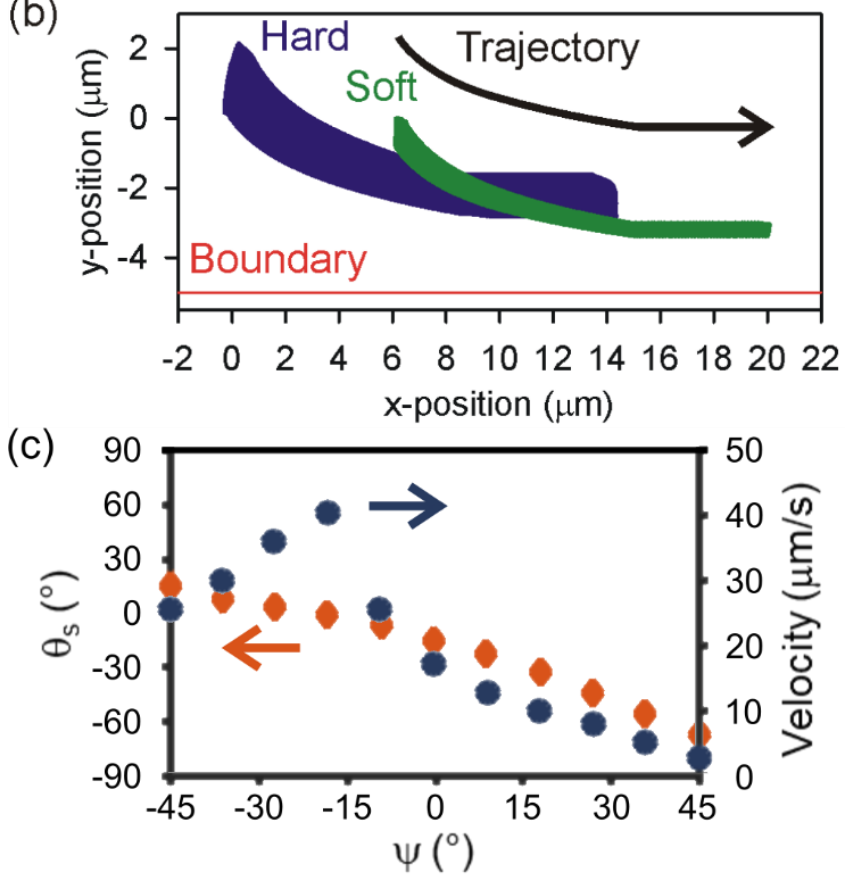

Figure 2: Traces of individual bead motion (a) in an unconstrained system and (b) when a single surface boundary is placed at $y=-5 \mu \mathrm{m}$. In each case, swimmers are initialised with the hard particle at $(0,0,0)$ and soft particle at $(6.5,0,0)$. The trajectory arrows are a guide to the eye indicating the overall propagation direction of the swimmer at elliptical field orientation $\psi=0^{\circ}$. (c) The effect of $\psi$ for a single surface boundary on the swimmer orientation, $\theta_{s}$, (red diamonds) and the final propagation velocity (blue circles). Details of the traces shown in (a) and (b), in which motion during the final three field cycles are resolved, are presented in Supplementary Material figures SM1 and SM2, respectively. 

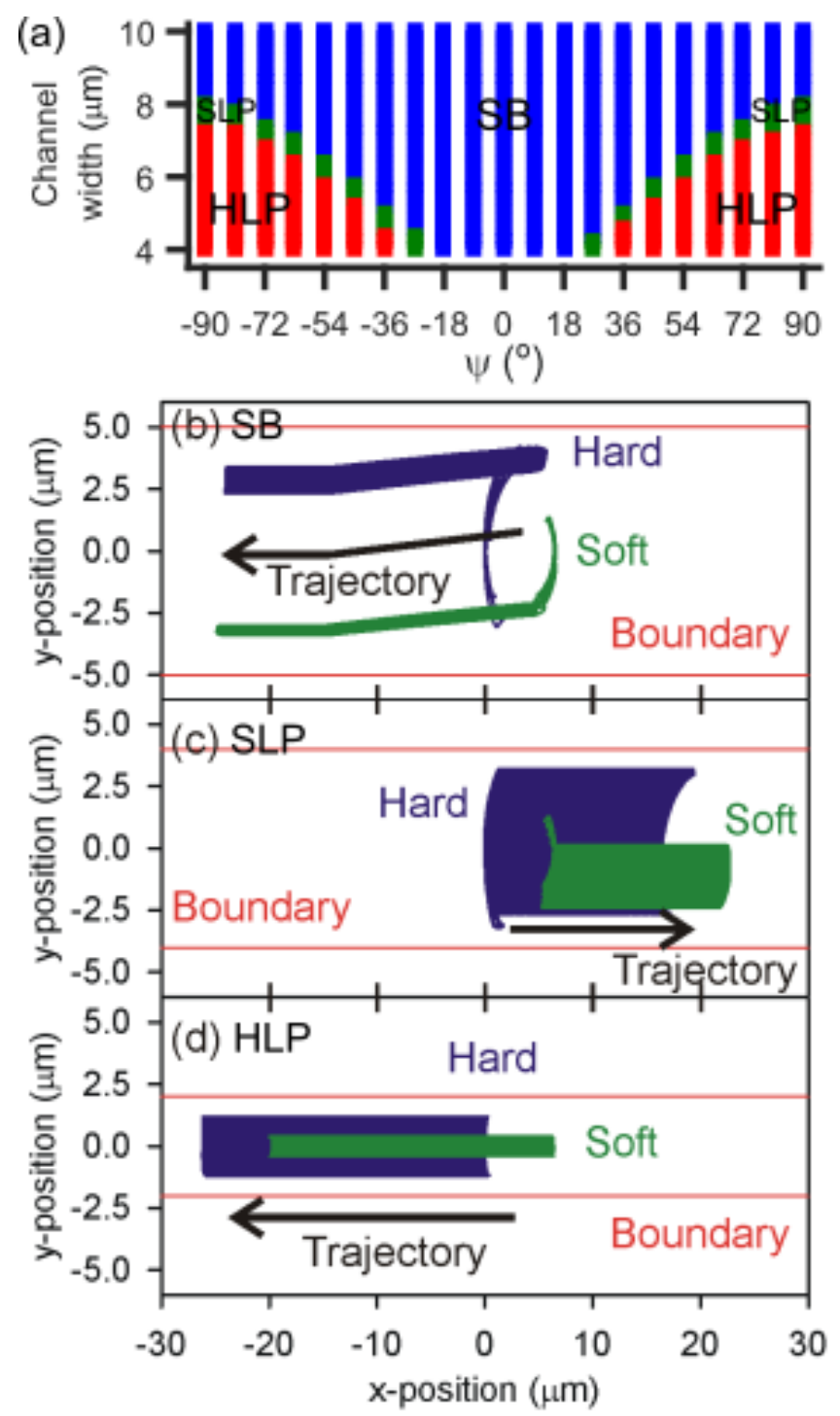

Figure 3: (a) Phase diagram of the channel propagation modes: single boundary (SB, blue), soft-leading pendulum (SLP, green) and hard-leading pendulum mode (HLP, red). (b-d) Example traces of individual bead motion during each propagation mode when $\psi=90^{\circ}$ for channel widths of (b) $10 \mu \mathrm{m}$, (c) $8 \mu \mathrm{m}$ and (d) $4 \mu \mathrm{m}$. Swimmers are initialised with the hard particle at $(0,0,0)$ and soft particle at $(6.5,0,0)$. Trajectory arrows are a guide to the eye indicating the overall direction of travel. Details of the traces shown in (b), (c) and (d) in which motion during the final three field cycles are resolved, are presented in Supplementary Material figures SM3, SM4 and SM5, respectively. 


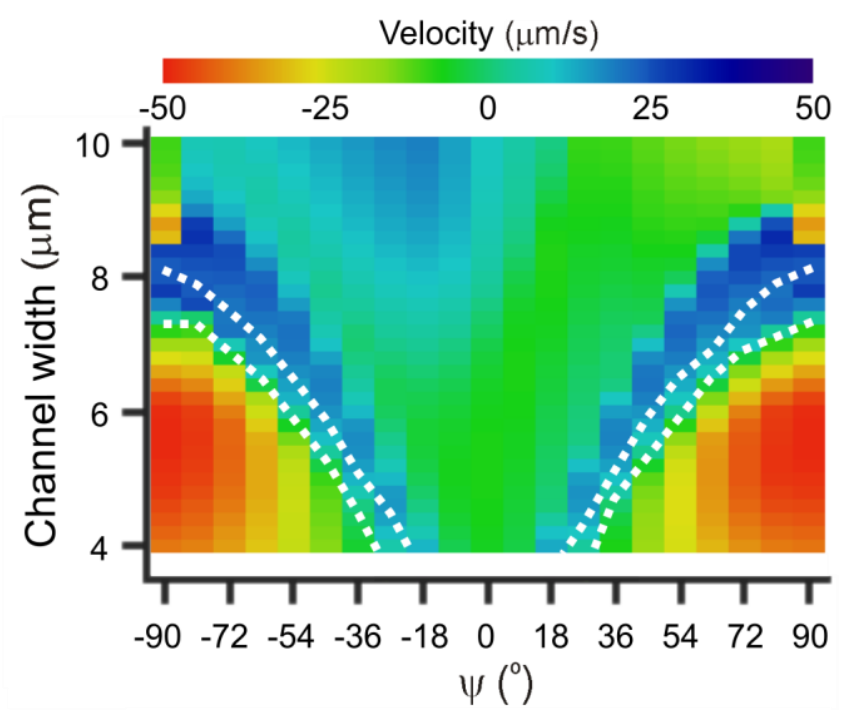

Figure 4: Heat map showing the dependence of the x-axis swimming velocity on the channel width and angle between the field major axis and the channel plane over a $3 \mathrm{~s}$ period. Dotted lines outline the phase boundaries of the propagation modes shown in fig. $3 a$.

\section{References}

J. G. Gibbs and P. Fischer, Chem. Commun. 51, 4192 (2015).

K. J. Rao, F. Li, L. Meng, H. R. Zheng, F. Y. Cai, and W. Wang, Small 11, 2836 (2015).

N. Bowden, M. T. Bryan, H. Duckles, S. Feng, S. Hsiao, H. R. Kim, M. Mahmoud, B. Moers, J. Serbanovic-Canic, I. Xanthis, V. C. Ridger, and P. C. Evans, Antioxid. Redox Signal. 25, 389 (2016).

M. Tanase, E. J. Felton, D. S. Gray, A. Hultgren, C. S. Chen, and D. H. Reich, Lab Chip 5, 598 (2005).

T. Qiu, T. C. Lee, A. G. Mark, K. I. Morozov, R. Munster, O. Mierka, S. Turek, A. M. Leshansky, and P. Fischer, Nat. Commun. 5, 5119 (2014).

S. T. Chang, V. N. Paunov, D. N. Petsev, and O. D. Velev, Nat. Mater. 6, 235 (2007).

S. J. Ebbens and J. R. Howse, Soft Matter 6, 726 (2010).

S. Palagi, A. G. Mark, S. Y. Reigh, K. Melde, T. Qiu, H. Zeng, C. Parmeggiani, D. Martella, A. Sanchez-Castillo, N. Kapernaum, F. Giesselmann, D. S. Wiersma, E. Lauga, and P. Fischer, Nat. Mater. 15, 647 (2016). S. Tottori, L. Zhang, F. Qiu, K. K. Krawczyk, A. Franco-Obregon, and B. J. Nelson, Adv. Mater. 24, 811 (2012). W. Gao, X. Peng, A. Pei, C. R. Kane, R. Tam, C. Hennessy, and J. Wang, Nano Lett. 14, 305 (2014).

A. Acemoglu and S. Yesilyurt, Microfluid. Nanofluidics 19, 1109 (2015).

M. Medina-Sanchez, L. Schwarz, A. K. Meyer, F. Hebenstreit, and O. G. Schmidt, Nano Lett. 16, 555 (2016).

A. Barbot, D. Decanini, and G. Hwang, Sci. Rep. 6, 19041 (2016).

A. Snezhko and I. S. Aranson, Nat. Mater. 10, 698 (2011).

G. Grosjean, G. Lagubeau, A. Darras, M. Hubert, G. Lumay, and N. Vandewalle, Sci. Rep. 5, 16035 (2015).

F. Martinez-Pedrero, A. Ortiz-Ambriz, I. Pagonabarraga, and P. Tierno, Phys. Rev. Lett. 115, 138301 (2015).

F. Martinez-Pedrero and P. Tierno, Phys. Rev. Appl. 3, 051003 (2015).

A. D. Gilbert, F. Y. Ogrin, P. G. Petrov, and C. P. Winlove, Q. J. Mech. Appl. Math. 64, 239 (2011). 
F. Y. Ogrin, P. G. Petrov, and C. P. Winlove, Phys. Rev. Lett. 100, 218102 (2008).

Q. A. Pankhurst, N. K. T. Thanh, S. K. Jones, and J. Dobson, J. Phys. D-Appl. Phys. 42, 224001 (2009).

Q. A. Pankhurst, J. Connolly, S. K. Jones, and J. Dobson, J. Phys. D-Appl. Phys. 36, R167 (2003).

R. Dreyfus, J. Baudry, M. L. Roper, M. Fermigier, H. A. Stone, and J. Bibette, Nature 437, 862 (2005).

M. T. Bryan, H. Duckles, S. Feng, S. T. Hsiao, H. R. Kim, J. Serbanovic-Canic, and P. C. Evans, Arterioscler. Thromb. Vasc. Biol. 34, 2199 (2014).

G. Grosjean, M. Hubert, G. Lagubeau, and N. Vandewalle, Phys. Rev. E 94, 021101 (2016).

G. C. Pereira and K. Zoltan, J. Biomed. Eng. Med. Devic. 2, 117 (2016).

D. Pimponi, M. Chinappi, P. Gualtieri, and C. M. Casciola, J. Fluid Mech. 789, 514 (2016).

A. J. T. M. Mathijssen, T. N. Shendruk, J. M. Yeomans, and A. Doostmohammadi, Phys. Rev. Lett. 116, 028104 (2016).

J. R. Blake, Proc. Camb. Phil. Soc. 70, 303 (1971).

A. D. Gilbert, F. Y. Ogrin, P. G. Petrov, and C. P. Winlove, Eur. Phys. J. E 34, 121 (2011). 